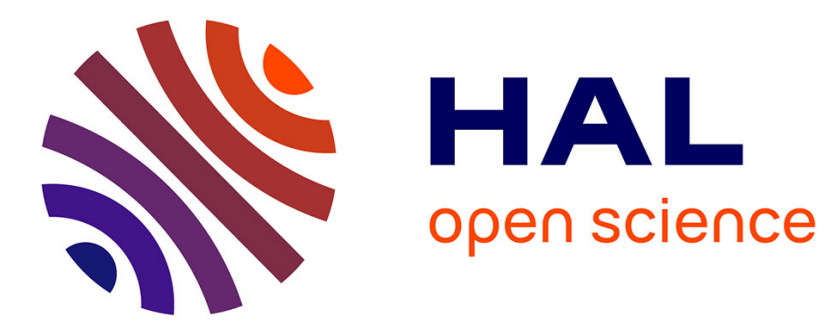

\title{
Les enceintes fortifiées de l'âge du Fer dans le Finistère
}

Patrick Maguer

\section{To cite this version:}

Patrick Maguer. Les enceintes fortifiées de l'âge du Fer dans le Finistère. Bulletin de l'Association française pour l'étude de l'âge du fer, 1995, 13, pp.41-42. hal-02549059

\section{HAL Id: hal-02549059 \\ https://hal.science/hal-02549059}

Submitted on 21 Aug 2020

HAL is a multi-disciplinary open access archive for the deposit and dissemination of scientific research documents, whether they are published or not. The documents may come from teaching and research institutions in France or abroad, or from public or private research centers.
L'archive ouverte pluridisciplinaire HAL, est destinée au dépôt et à la diffusion de documents scientifiques de niveau recherche, publiés ou non, émanant des établissements d'enseignement et de recherche français ou étrangers, des laboratoires publics ou privés.

\section{(이)(\$)}

Distributed under a Creative Commons Attribution - NonCommercial - NoDerivatives| 4.0 


\section{Les enceintes fortifiées de l'Age du Fer dans le Finistère}

L'expédition britannique de 1938, dirigée par Sir M. Wheeler, a recensé dans le département du Finistère plus d'une centaine d'enceintes fortifiées de diffèrentes périodes. Les informations recueillies dans les cahiers, constitués lors de ces prospections, ont permis de reprendre le recensement de ces structures afin d'en déterminer les caractéristiques morphologiques et leur organisation spatiale au sein du département. L'examen des cahiers inédits Wheeler, complété par différentes sources, a fait ressortir 38 sites dont la fréquentation remonterait à l'Age du Fer.

\section{Les facteurs d'implantation}

le relief:

Les fortifications sont majoritairement implantées sur des promontoires ou des éperons délimités soit par l'océan, soit par la confluence de deux cours d'eau, ou bien sont présentes sur des rebords de sommets de colline et de plateau. Le relief est en relation étroite avec le type morphologique : les enceintes de contour sont situées là où le relief est le mieux marqué (chaînes des Monts d'Arrée, région de Quimper), les fortifications quadrangulaires étant préférentiellement implantées sur des zones planes ( plateau, vastes sommets de colline).

\section{les matières premières:}

Il existe sans doute une corrélation entre les gisements de matières premières et certaines fortifications. C'est probablement le cas à Kerguily en Dineault où l'enceinte quadrangulaire est à l'emplacement d'un gite superficiel de minerai de fer. Les recherches menées au XIXè siècle sur le Camp d'Artus ont montré une exploitation ancienne des gisements de plomb argentifère aux abords de l'oppidum. Plus largement, la présence en milieu alluvionnaire de minerai d'étain peut être mise en relation avec une forte densité d'enceintes fortifiées, notamment dans le Léon et dans l'extrême sud du Finistère.

\section{les voies de communication :}

Le cas des estuaires du Goyen, de la Laita et de l'Odet est à ce titre tout à fait exemplaire. En effet, dans les trois cas, nous avons noté la présence systématique de deux fortifications là où la rivière forme un coude et rencontre un affluent. Ces enceintes sont soit sur des rives opposées, soit sur une même rive mais séparées par l'affluent. Les voies maritimes sont illustrées par la présence de fortifications sur des portions côtières aménageables en port où en zone d'échouage (presquîle de Kermorvan, Le Conquet; pointe de Primel Tregastel, Plougasnou ; pointe de Kastell Ac'h, Plouguerneau).

\section{Typomorphologie et surface}

Sur les 38 enceintes répertoriés, 15 sont des barrages d'éperon et de promontoire, 13 des fortifications quadrangulaires, 4 des enceintes fortifiées de contour, un cas possible 
d'enceintes accolées et 5 de morphologie indéterminée. Les surfaces, généralement peu importantes, sont en relation étroite arec le type morphologique. Les superficies les plus importantes correspondent aux enceintes de contour et aux barrages d'éperon et de promontoire. Sur 31 cas dont les dimensions sont connues, seulement 12 sites ont une surface supérieure ou égale à 2.00 ha et il n'existe pas de groupe intermédiaire entre les très grandes fortifications (plus de 20.00ha) et les établissements de moyenne superficie (entre 2.00 et 4.50ha).

\section{Les structures défensives et les aménagements internes et de proximité}

Les remparts sont majoritairement constitués par une accumulation de pierres et de terre, mais avec parfois des aménagements plus soignés sous la forme de parements externe et interne, et dans deux cas au moins la présence de poutrage en bois (murus gallicus du Camp d'Artus, rempart partiellement vitrifié de l'éperon barré de Beg-ar-Castel). La multivallation des fortifications est visible surtout sur les éperons barrés en raison du substrat rocheux sous-jacent qui rend difficile le creusement de fossé défensif. C'est la largeur de l'ensemble qui détermine dans ce cas la valeur défensive du vallum. Ce type de défense est également présent sur trois enceintes de contour et quelques cas ont pu être reconnus sur des fortifications quadrangulaires.

Les structures internes et de proximité sont dans la plupart des cas des souterrains de l'Age du Fer ou des structures funéraires dont des tumuli. Quelques cas de structures excavées sont répertoriés sur des sites côtiers (Castel-Meur, Castel $\mathrm{Coz}$ au Cap Sizun ; pointe de Lostmac'h dans la presqu'île de Crozon).

\section{Distribution de l'habitat fortifié dans le Finistère}

Outre la méthode des polygones de Thiessen qui met en évidence onze sous-ensembles et des zones de forte densité de l'habitat fortifié, nous avons procédé à une analyse de cartographie comparée des enceintes avec les souterrains et les ateliers de briquetage. Il a ainsi été possible de définir 7 micro-territoires qui se caractérisent par des concentrations de souterrains autour de une à trois fortifications de plus de 2.00 ha. Ces sous-groupes suggèrent une organisation de l'espace en petites unités dominées par une ou plusieurs enceintes dont la vocation militaire et politique est associée à d'autres activités comme l'agriculture, l'artisanat et le commerce. La présence, à l'intérieur de ces mêmes unités, de plusieurs établissements fortifiés laisse supposer qu'il existe des fonctions complémentaires et une hiérarchisation de ces ensembles. 